\title{
Corrigendum
}

\section{Schwerpunkt: Nietzsches genealogische Methode: Historismus, Relativismus und Anthropologie}

\author{
https://doi.org/10.1515/dzph-2021-9999
}

Corrigendum zu: Schwerpunkt: Martin Kusch, Markus Wild. September 2019. Nietzsches genealogische Methode: Historismus, Relativismus und Anthropologie. Deutsche Zeitschrift für Philosophie. Band 67, Heft 3, S. 414-417 (DOI: https:// doi.org/10.1515/dzph-2019-0032)

In dem in Heft 3/2019 publizierten Themenschwerpunkt zu „Nietzsches genealogischer Methode“ ist es leider zu einem Zitationsfehler gekommen. In der Fußnote 5 zur Einleitung werden Craig (1980), Williams (2002) und Joas (2011) als Vertreter verschiedener Formen von affirmativer Genealogie genannt. Durch die chronologische Reihung in der Anmerkung sollte nicht der Eindruck erweckt werden, Joas bezöge sich auf Craig oder Williams. Hans Joas hat den Ausdruck „affirmative Genealogie“ geprägt und wir verwenden den Ausdruck in einem weiteren Sinne zur deutschen Übersetzung der im Englischen üblichen Rede von einer „vindicatory genealogy“. Gleiches gilt für die Verwendung von „affirmativer Genealogie“ in den Beiträgen von Katharina Kinzel und Matthieu Queloz. Hier ist der ausdrückliche Hinweis auf Joas unterblieben, da beide sich primär auf die „vindicatory genealogy“ beziehen und der Auffassung waren, dass der Hinweis in der Einleitung als Verweis auf eine maßgebliche Quelle im deutschsprachigen Raum genügt. Dabei wurde aber übersehen, dass Einleitung und Einzelbeiträge auch unabhängig voneinander gelesen werden können.

Für die Gastherausgeber und Autor:innen

Markus Wild

Martin Kusch, martin.kusch@univie.ac.at

Markus Wild, markus.wild@unibas.ch 